\title{
Exploring the structural stability order and electronic properties of transition metal M@Ge12 (M = Co, Pd, Tc, and Zr) doped germanium cage clusters
}

\author{
Ravi Trivedi ${ }^{1}$ and Vikash Mishra ${ }^{1}$ \\ ${ }^{1}$ Indian Institute of Technology Bombay
}

August 28, 2020

\begin{abstract}
In the present report, the structural stability order and electronic properties of the transition metal M@Ge12 $(\mathrm{M}=\mathrm{Co}, \mathrm{Pd}$, Tc, and $\mathrm{Zr}$ ) doped germanium cage has been carried out at B3LYP/LANL2DZ ECP level by using spin polarized density functional theory. Initially, we selected five lowest energy structure of neutral TM doped Ge12 cluster with high symmetry point like D6h-symmetric hexagonal prism (HP), the D6d-symmetric hexagonal anti-prism (HAP), D2d-symmetric bi-capped pentagonal prism (BPP), perfect icosahedrons (Ih) and Fullerene type structures. Further, we discussed the electronic origin of stability as well as electronic properties by calculating binding energy, HOMO-LUMO gap, charge transfer mechanism and density of states. We indentified that the Pd, Tc, and Zr encapsulated Ge12 cage with hexagonal prism [HP] structures are minimum energy structures while Co@Ge12 cage prefer HAP structure. The magnitudes of binding energy of the clusters indicate that the doping of $4 \mathrm{~d}$ transition metal gives most stable structure rather than $3 \mathrm{~d}$ transition metal Co atom. The large HOMO-LUMO gap and natural bond orbital analysis explain the stability of these clusters using closed shell electronic configuration and the contribution of $\pi$ and $\sigma$ bond. Charge transfer mechanism shows that the Tc, Pd and Zr atoms play role as an electron donor in the system whereas Co inclined to accept the electrons. The importances of "d" orbital in localized electrons near the Fermi level are also explained through partial density of states.
\end{abstract}

\section{Keywords -}

Binding energy, HOMO-LUMO gap, Density of states, Charge analysis, Density functional theory

\section{Introduction}

Electronic and structural properties of transition metal encapsulated in germanium clusters are incredibly dynamic area of exploration because of its significant in building block for clusters assembled materials and other expected applications in numerous fields ${ }^{[1-10]}$. Without a doubt, doping in silicon confine clusters has additionally stood out because of its applications in nanoelectronic gadgets and building blocks nanomaterials ${ }^{[7-10]}$. The decision of various sort of transition metal molecules prompts the much attractive legitimacy in the properties of these confine clusters. It has additionally been researched that these hybrid nano clusters could be collected to frame nanotubes ${ }^{[11-12]}$. As we probably aware, germanium has predominant electron and opening mobilities ${ }^{[13-14]}$ because of the less viable mass as opposed to silicon, so germanium is perhaps the most option in contrast to silicon in the field of semiconductor nanomaterials. Although, pure germanium semiconductor clusters are chemically reactive due to the presence of dangling bond ${ }^{[15-16]}$. Its mean reactive Ge cage can be stabilized by the doping of transition metal atom likewise to the instance of TM-doped silicon groups ${ }^{[17-18]}$. Metal doped germanium groups play distinctive growth behavior and electronic properties from the metal doped silicon clusters ${ }^{[19-22]}$. The experimetnal examination on TM metal doped $\mathrm{Si}, \mathrm{Ge}, \mathrm{Sn}$ and $\mathrm{Pb}$ groups show that the stabilities qualities are identified with development of 
enclosure like structure just as both host and dopant particles ${ }^{[23]}$. The transition metal doping in germanium confine clusters give a one of a kind medium to investigating new auxiliary and electronic properties rely upon the group size and doping ${ }^{[1-4]}$. In light of our past report ${ }^{[1-4,12-15,16]}$ on TM metal doping germanium nanoclusters by utilizing density fnctional theory (DFT) concentrate on unadulterated germanium clusters found that $\mathrm{Ge}_{10}$ (icosahedral), $\mathrm{Ge}_{12}$ (Hexagonal crystal) and $\mathrm{Ge}_{16}$ (Fullerene or Frank-kesper) structures are exceptionally stable hollow clusters groups with a huge inner volume proposing conceivable endohedral doping to frame another class of hybrid nanoclusters with tuned properties. In these three structures, we concentrated on $\mathrm{Ge}_{12}$, hexagonal crystal structure, which is the most contemplated species recommending that metal molecule immerses the valence electrons of twelve germanium particles by sitting in the centre point of the ring. In view of present hypothesis, we showed a noteworthy $\mathrm{D}_{6 \mathrm{~h}}$ symmetric hexagonal crystal (HP) ground state structure for $\mathrm{M} @ \mathrm{Ge}_{12}(\mathrm{M}=\mathrm{Co}, \mathrm{Pd}, \mathrm{Tc}$, and $\mathrm{Zr})$. Recently our group ${ }^{[2]}$ found the role of shell closing model and NICS in the stability of $\mathrm{Nb}$ doped germanium group inside the size range of $\mathrm{n}=7-18$ germanium atom and anticipated hexagonal crystal type geometry is ground state structure. Electronic and optical properties of $\mathrm{Ag}$ and $\mathrm{Au}$ doped Gen $(\mathrm{n}=10,12 \text {, and } 14)^{[3]}$ groups detailed a $\mathrm{D}_{6 \mathrm{~h}}$ hexagonal crystal singlet ground state structure. Thermodynamical and synthetic soundness of Mo@Ge 12 group in the size scope of Ge from 1 to 20 and legitimacy of 18 electron counting rule from the conduct of various determined boundaries has explored by the trivedi et al. ${ }^{[4]}$ and they found the hexagonal crystal has least energy in the arrangement.

As a past report on other TM metal doped germanium work, the structure of $\mathrm{Ni}_{\mathrm{G}} \mathrm{Ge}_{12}$ hybrid cluster has a pseudo-icosahedral triplet, ${ }^{[24]}$ a D2d-symmetric singlet ${ }^{[25]}$ or a puckered hexagonal prismatic singlet ${ }^{[26]}$ (BLYP, B3PW91 or PW91 functionals, separately). So also, icosahedral sextet ${ }^{[27]}$ and hexagonal prismatic

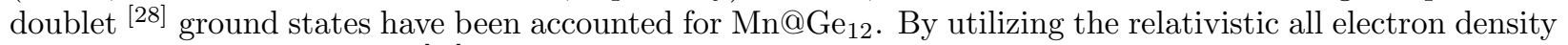
functional theory Tang et. al ${ }^{[24]}$ revealed the structure, solidness and electronic properties of TM@Ge 12 [TM - Sc to Ni). It was discovered that all the custers are maybe incompletely metallic and the ground state structure is most likely icosahedron. V. kumar et al. ${ }^{[29]}$ contemplated the $\mathrm{ZnGe}_{12}$ metal typified superatom, in which they found that doping of $\mathrm{Zn}$ in germanium created icosahedron ground state structure. Thus, metal doped germanium clusters MGen at the size of $n=10,12$ researched by J. Lu and S. Nagase [30].

In this current report, we break down the size stability and electronic properties of $\mathrm{M}_{\mathrm{G}} \mathrm{Ge}_{12}(\mathrm{M}=\mathrm{Co}$, $\mathrm{Pd}, \mathrm{Tc}$, and $\mathrm{Zr}$ ) nanoclusters. Electronically Tc is described considerably field $4 \mathrm{~d}^{5}$ cell joined with $5 \mathrm{~s}^{2}$ valence cell. Co and Zr, then again show $3 \mathrm{~d}^{7}$ and $4 \mathrm{~d}^{2}$ " $\mathrm{d}$ " orbital setups joined with $4 \mathrm{~s}^{2}$ and $5 \mathrm{~s}^{2}$ valence cell individually. Essentially, Pd molecule has a field $4 \mathrm{~d}^{10}$ cell joined with $5 \mathrm{~s}^{0}$ valence electron. Since the adjustment of dopant embodied germanium confine firmly relies upon the $\mathrm{d}$ band filling. The empty d orbital can oblige the dangling bonds on confine surface and give a solid strong interaction among dopant and have confine. These current arrangements of transtion metal atoms were chosen based on the development they can give to tune the properties of germanium confine groups. The advancement is accomplished in the TM metal doped Ge confine groups yet at the same time there are some inquiries for academic network that there is no immediate experimetnal check on the soundness order of $3 \mathrm{~d}$ and $4 \mathrm{~d}$ TM metal doped germanium confine clusters. The host germanium confine are vacant and numerous TM doped molecule could be utilized as dopant to shape new endohedral group that would especially show new electronic and thermodynamic properties that are unique in relation to the unadulterated germanium clusters.

\section{Computational method}

The calculations to search low lying structures of $\mathrm{M}_{\mathrm{G}} \mathrm{Ge}_{12}(\mathrm{M}=\mathrm{Co}, \mathrm{Pd}, \mathrm{Tc}$, and $\mathrm{Zr})$ in this work began with lot of previous geometries where transition metal atom sits at various different position i.e. (1) Substitution (2) Endohedral (3) Exohedral on the basis of optimized $\mathrm{Ge}_{12}$ and calculated at all possible spin states as reported in the literature ${ }^{[1-10]}$. All the initial geometries optimized without any symmetry constraint. For the optimization [to get total minimum energy], we used B3LYP [31-34] exchange correlation function with spin polarized generalized gradient approximation (GGA) as implemented in the Gaussian 03 computational code ${ }^{[35]}$ which is based on linear combination of atomic orbitals density functional theory method. A very standard Gaussian basis (LANL2DZ) sets coupled with effective core potential to express molecular orbital 
as linear combination of atom-centered basis function is used on all atoms. This basis set can reduce the difficulties in two electron integrals caused by the doped transition metal atoms ${ }^{[36-39]}$. We have used $3 \mathrm{~d}^{7}$ $4 \mathrm{~s}^{2}, 4 \mathrm{~d}^{10} 5 \mathrm{~s}^{0}, 4 \mathrm{~d}^{5} 5 \mathrm{~s}^{2}$, and $4 \mathrm{~d}^{2} 5 \mathrm{~s}^{2}$ configuration for $\mathrm{Co}, \mathrm{Pd}, \mathrm{Tc}$, and $\mathrm{Zr}$ and $4 \mathrm{~s}^{2} 4 \mathrm{p}^{2}$ configuration for Ge respectively. The accuracy of standard Gaussian basis (LANL2DZ) sets for different transition metal atoms doped in germanium cage clusters was validated by many recently publications ${ }^{[2-4,15-17]}$. In present work, the minimum energy structure accepted as optimized when the maximum displacement of atoms, RMS displacement of atoms and the maximum force of atoms have very less magnitudes respectively. Furthermore, we have also corrected the zero point energy correction of the isomer however they are not expected to affect the relative binding energy [40]. The ground state structures were calculated at the same level of theory and found zero imaginary frequency to make sure that the optimized geometries corresponds to real local minima. To find the nature of materials and localized and delocalized electrons near the Fermi level, we also obtained the partial density of states (PDOS) using GaussSum software ${ }^{[41]}$. The natural bond orbital analysis (NBO) analysis ${ }^{[42-43]}$ was also conducted to find out the charge analysis of valence orbitals on each atom and contribution near the Fermi level in the DOS.

Further to check the quality of our adopted method, test calculations were performed on the Ge-Ge and Ge-Co, Ge-Pd, Ge-Tc, and Ge-Zr dimmers. The bond length and frequencies of these dimmers are $2.54 \AA$ $\left(245 \mathrm{~cm}^{-1}\right), 2.26 \AA\left(273 \mathrm{~cm}^{-1}\right), 2.25 \AA\left(307 \mathrm{~cm}^{-1}\right), 2.25 \AA\left(291 \mathrm{~cm}^{-1}\right)$, and $2.44 \AA\left(307 \mathrm{~cm}^{-1}\right)$ respectively. All the calculated structural parameter such as the bond length and frequency compared with other theoretical and experimental results shown in table 1 . To further check the validity of our functional we determined bond length and frequency with different functional such as (B3LYP, MPW1PW91, and B3PW91). All the related parameters are shown in table 2. The outcomes gained by the B3LYP functional with LANL2DZ ECP basis set are acceptable as it is in good agreement with reported theoretical and experimental results [1-4, 44-49]

\section{Results and discussion}

Five significant kind of introductory structure of neutral $\mathrm{Ge}_{12}$ cluster with high symmetry point are appeared in figure 1 . To decide the most minimal energy structure of unadulterated $\mathrm{Ge}_{12}$ cluster, we chose 5 separate high symmetry geometries: the $\mathrm{D}_{6 \mathrm{~h}}$-symmetric hexagonal crystal $(\mathrm{HP})$, the $\mathrm{D}_{6 \mathrm{~d}}$-symmetric hexagonal anti-prism (HAP), $\mathrm{D}_{2 \mathrm{~d}}$-symmetric bi-capped pentagonal prism (BPP), immaculate icosahedrons $\left(\mathrm{I}_{\mathrm{h}}\right)$ and Fullerene type structures. We improved these underlying geometries at B3LYP/LANL2DZ ECP level of theory.

\section{Effect of TM doping}

So as to talk about the impacts of transition metal doped impurity on pure $\mathrm{Ge}_{12}$ confine clusters, we did again least energy optimization calculation by utilizing strategy referenced in computational methodology segment. The 12-vertex TM metal doped cage clusters are extraordinary in three totally various geometries like hexagonal crystal [HP], Icosahedral [IH] or bicapped pentagonal prism [BPP] among the five endohedral structure characterized as in figure 1. It must be brought up that $\mathrm{Ge}_{12}$ confine cluster lean toward hexagonal crystal structure with $\mathrm{TM}=\mathrm{Tc}, \mathrm{Pd}$, and $\mathrm{Zr}$, comparable as some past reports ${ }^{[1-4,7,16,20]}$ aside from Co doping which has hexagonal anti-prism [HAP] symmetry.

Noteworthy twisting from the high symmetry limits after relaxation because of the $\mathrm{TMGe}_{12}$ confine local interaction characterized the Jahn teller ${ }^{[50]}$ impact that can be found in the figure 2 . The ground state structures with some metastable isomers having diminishing size dependability order in optimized structure with energy contrast among them are appeared in figure 2. Utilizing present approach, we got hexagonal anti-prism [HAP] symmetry with 57 valence electrons is more steady than hexagonal-crystal [HP] structure in $\mathrm{Ge}_{12} \mathrm{Co}$ cluster. The energy distinction is extremely less around $0.002 \mathrm{eV}$ only. Our predicted outcome is totally different with icosahedral dependability of first line transition metal $(\mathrm{Zn}, \mathrm{Mn})^{[28,30]}$ atom doped $\mathrm{Ge}_{12}$ confine. The complete "d" electron count including all valence "nd" electrons on $\mathrm{M} @ \mathrm{Ge}_{12}(\mathrm{M}=\mathrm{Co}$, $\mathrm{Pd}, \mathrm{Tc}$, and $\mathrm{Zr})$ is determined as $[12 * 4+(9,10,7,4)=57,58,55,52]$ all through this manuscript. The other optimized structures as BPP, Ih, and Fulla are $0.54 \mathrm{eV}, 0.79 \mathrm{eV}$, and $1.65 \mathrm{eV}$ are not exactly stable HAP 
symmetry separately. The strength of $\mathrm{CoGe}_{12}$ is additionally rely upon 18 electron including rule as studied in $\mathrm{Ni} @ \mathrm{Ge}_{12}$ by Bandyopadhyay et al ${ }^{[25]}$. As we probably aware if there are eight electrons in external electronic shells like $\mathrm{ns}^{2} \mathrm{np}^{6}$, the primary group elements are steady, while 18 electron counting rule is identified with transition metal doping with $\mathrm{Si}$ and Ge confine, which having most extreme strength and follow the closed shell of $\mathrm{ns}^{2}(\mathrm{n}-1) \mathrm{d}^{10} \mathrm{np}^{6}{ }^{[51-54]}$. The valence electrons in Co atom are $3 \mathrm{~d}^{7} 4 \mathrm{~s}^{2}$ means all out 9 electrons yet because of strong interaction among Co and $\mathrm{Ge}_{12}$ confine the 8 "d" orbitals are for the most part contributing as should be obvious in the table1 in valuable data. Because of this association the cage system adhering to 18 electrons counting rules.

Then again the ground state structure of $\mathrm{PdGe}_{12}$ again puckered hexagonal prism with a $\mathrm{Pd}$ atom, involve endohedral position in the middle of two hexagons of germanium atom like our past outcomes on $\mathrm{Nb}$ and Mo doped germanium clusters ${ }^{[2,4]}$. We found that the triplet state is more stable instead of singlet multiplicity utilizing the B3LYP functional in $\mathrm{Pd} @ \mathrm{Ge}_{12}$ clusters. The total valence electrons include in this framework are 58 which is magic number. TM metal doped germanium small cluster show soundness at electron count $2,8,18,20,34,40,58 \ldots$ separately known as superatom and it is affirmed experimentally likewise ${ }^{[55]}$. For this situation, the degenaracies in electronic shells can be evacuated by geometrical mutilation and stabilize the clusters. So $\mathrm{Pd} @ \mathrm{Ge}_{12}$ can be a superatom with $2 \mu \mathrm{B}$ magnetic moment as like $\mathrm{MnGe}_{12}$ or $\mathrm{MnSn}_{12}$ as revealed beforehand ${ }^{[56]}$. The 58 valence electrons designed in superatomic shell as $1 \mathrm{~S}^{2} 1 \mathrm{P}^{6} 1 \mathrm{D}^{8} 1 \mathrm{~F}^{6} 1 \mathrm{G}^{2} 2 \mathrm{D}^{6} 2 \mathrm{~S}^{2} 1 \mathrm{D}^{2} 1 \mathrm{~F}^{2} 1 \mathrm{G}^{8} 1 \mathrm{~F}^{4} 1 \mathrm{G}^{8} 2 \mathrm{D}^{4}$. [Molecular orbital energy diagram has also shown in supplementary information as SF1] Presently on the off chance that we consider the Tc and Zr particle in germanium confine clusters as introduced in figure 2, out of five advanced geometries the hexagonal crystal structure in singlet spin state is ground state. In some report, the TM doped germanium fullerene like structure which is combination of four pentagon and four rhombi and icosahedrons structure show ground state $^{[19,7]}$. Be that as it may, it not in every case genuine on the grounds that base energy structure relies upon doping as model the Ti, Zr, Hf, Nb, Mo, and Zn doped fullerene or icosahedrons structure isn't a ground state structure for $\mathrm{n}=12$. Past examination shows that metal exemplified $\left[\mathrm{M} @ \mathrm{Ge}_{12}\right.$, where $\mathrm{M}=\mathrm{Ti}$, $\mathrm{Zr}, \mathrm{Hf}, \mathrm{Nb}, \mathrm{Mo}$, and Zn] hexagonal prism is demonstrated to be least vitality structure $[2,4,15-16,30]$. The hexagonal anti-prism and icosahedral both are $0.001 \mathrm{eV}$ and $1.63 \mathrm{eV}$ lower than ground state structure where as in $\mathrm{Zr}$ case the icosahedral and hexagonal enemy of crystal [HAP] both are $0.29 \mathrm{eV}$ and $1.02 \mathrm{eV}$ lower than hexagonal crystal structure. The orbital compositions of every atom in our system has additionally determined utilizing Multi-wave function ${ }^{[57]}$ and appeared in table 3 in supplementary information.

In view of this geometrical investigation of $\mathrm{TM} @ \mathrm{Ge}_{12}[\mathrm{TM}=\mathrm{Co}, \mathrm{Pd}, \mathrm{Tc}$, and $\mathrm{Zr}]$ clusters and correlation of our anticipated outcomes with past theoretical and experimental results, we can sum up many intriguing patterns as follows:

1. Contrasting and unadulterated Ge clusters, The TM metal doping consistently prompts structure recreation. If we see different past examination ${ }^{[1-4,6-10]}$ of TM metal doped Ge groups, the TM metal atom like to take position from exohedral to endohedral as the size fluctuates from $n=1-20$. As the size increment implies n [?] 9, the TM molecules totally fall into the germanium confine.

2. We can distinguish that the $\mathrm{D}_{6 \mathrm{~h}}$ symmetrical hexagonal prism structure is interesting for germanium just as silicon confine as portrayed by José M. Goicoechea et al. in their examination ${ }^{[58]}$. It tends to be found in figure 2, the HP, Ih, and HAP structure arrive at their most extreme dependability when all out valence electron in the system have 52-58 electrons.

\section{Electronic Properties}

In this piece of our proposed work, we will talk about the electronic properties of germanium confine containing a transition metal atom dependent on determined (a) average binding energy [BE], (b) HOMO-LUMO gap and charge transfer mechanism and (c) partial density of states.

Binding energy - To get knowledge about the relative stability of clusters we calculate the average binding energy of the $\mathrm{TM} @ \mathrm{Ge}_{12}[\mathrm{TM}=\mathrm{Co}, \mathrm{Pd}, \mathrm{Tc}$, and $\mathrm{Zr}]$ clusters. The average binding energy can be defined 
using below mathematical formation:

$$
B E=\frac{\left[E_{\mathrm{TM}}+12 \times E_{\mathrm{Ge}}-E_{T M G e 12}\right]}{n}
$$

Where $\mathrm{E}_{\mathrm{TM}}, \mathrm{E}_{\mathrm{Ge}}$ and $\mathrm{ETMGe}_{12}$ are the ground state energy of transition metal, germanium and TM doped germanium confine individually. Here "n" characterizes the total number of atoms in the cage. In our current work the $\mathrm{n}=13$ for all figuring as $[\mathrm{n}=12 \mathrm{Ge}$ and $1 \mathrm{TM}$ atom]. The binding energy, HOMO-LUMO gap, charge on TM metal particle, bond length of Ge-TM, relative energy $(\Delta \mathrm{E})$ appeared in table 3 . We analyze the binding energy of $\mathrm{TM} @ \mathrm{Ge}_{12}[\mathrm{TM}=\mathrm{Co}, \mathrm{Pd}, \mathrm{Tc}$, and $\mathrm{Zr}]$ clusters and found that the Tc metal encapsulated germanium confine gives most stable structure. The remaining are in the order of $\mathrm{Zr}>\mathrm{Pd}$ $>$ Co. The average binding energy of TM doped germanium cage cluster with the correlation of pure $\mathrm{Ge}_{12}$ cluster is appeared in figure 3 .

The magnitude of binding energy of the clusters gives the information about the strength of the chemical bonding in the clusters. The binding energy value of pure germanium cluster is $2.062 \mathrm{eV}$ in HP ring obtained using the present method is consistent with our previous reports ${ }^{[1-4]}$. The average binding energy values of TM encapsulated germanium cage are $2.45 \mathrm{eV}, 2.40 \mathrm{eV}, 2.25 \mathrm{eV}$, and $2.23 \mathrm{eV}$ for Tc, Zr, $\mathrm{Pd}$, and Co respectively. It means the doping of $4 \mathrm{~d}$ transition metal $\mathrm{Tc}$, and $\mathrm{Zr}$ gives most stable structure rather than 3d transition metal Co atom.

It tends to be seen that the doping of $3 \mathrm{~d}$ and $4 \mathrm{~d}$ transition metal in pure $\mathrm{Ge}_{12}$ cage cluster can improve the dependability of pure germanium cluster. The value of binding energy given by other research group [49] of Tc@Ge $\mathrm{Ge}_{12}$ is around $3.02 \mathrm{eV}$ with BPW91/LANL2DZ level of theory. Our worth is less a direct result of the utilizing ECP basis set and a reasonable B3LYP functional. Essentially, the binding energy value of $\mathrm{Zr}$ doped $\mathrm{Ge}_{12}$ cluster is well predictable with past reports ${ }^{[16]}$. All the determined parameters like average binding energy, HOMO-LUMO hole, bond length of Ge-TM, and the relative energy $(\Delta \mathrm{E})$ contrast between stable isomers are appeared in table 3.

Here, we have also calculated the binding energy for all other isomers presented in figure2. We can easily see the variation of binding energy as the structures are changing. The comparisons of all other isomers are shown in figure 4 . The biding energy of Co doped germanium atom in hexagonal prism [HP] and hexagonal anti-prism are nearly same but the icosahedral and bicapped pentagon prism both are relatively less stable with the difference of $0.04 \mathrm{eV}$ and $0.06 \mathrm{eV}$ respectively. On the other hand, in $\mathrm{Pd} @ \mathrm{Ge}_{12}$, the binding energy difference between HP and HAP geometries is around $0.02 \mathrm{eV}$. The difference is quite large in icosahedral case which is around $0.07 \mathrm{eV}$. Similarly if we see the case of Tc and $\mathrm{Zr}$ doped germanium cage clusters, the binding energy difference is more as we move towards HP-HAP-IH-BPP geometries. We can conclude that the symmetry stability depend on the $3 \mathrm{~d}$ and $4 \mathrm{~d}$ transition metal. In the case of $3 \mathrm{~d}$ transition metal, the metal encapsulated hexagonal anti-prism is stable geometries whereas in $4 \mathrm{~d}$ transition metal we get hexagonal prism as a minimum energy structure as we predicted in our previous reports ${ }^{[2,4]}$.

\section{HOMO-LUMO gap and Charge transfer mechanism -}

Molecular orbital analysis can provide very important information on electronic structure. The ability of molecules and clusters to participate in chemical reaction depends upon the energy gap which is known as HOMO [Highest Occupied Molecular Orbital] LUMO [Lowest Unoccupied Molecular Orbital] gap. The wide energy gap of clusters also decides the optical polarizability of the molecule ${ }^{[4]}$. A large value of HOMOLUMO gap always indicate the closed shell electronic configuration ${ }^{[1-4]}$ and the ability to take jump from lower state to higher state. The $\mathrm{E}_{\mathrm{gap}}$ of $\mathrm{TM}_{\mathrm{G}} \mathrm{Ge}_{12}[\mathrm{TM}=\mathrm{Co}, \mathrm{Pd}, \mathrm{Tc}$, and $\mathrm{Zr}]$ clusters are summarizes in table 3 .

The results show that doping of $4 \mathrm{~d}$ transition metal like $\mathrm{Tc}, \mathrm{Zr}$, and $\mathrm{Pd}$ have relatively large gap in compare to 3d transition metal Co. Here we obtain $1.96 \mathrm{eV}$ [ $\left.\mathrm{Tc} @ \mathrm{Ge}_{12}\right], 1.96 \mathrm{eV}\left[\mathrm{Pd} @ \mathrm{Ge}_{12}\right], 1.86 \mathrm{eV}\left[\mathrm{Zr} @ \mathrm{Ge}_{12}\right]$, and $0.97 \mathrm{eV}\left[\mathrm{Co} @ \mathrm{Ge}_{12}\right]$ HOMO-LUMO gap for the most stable clusters. The stability of these clusters can be 
defined using closed shell electronic configuration and the contribution of $\pi$ and $\sigma$ bond. The energy gap of Tc and Pd doped germanium cluster is biggest among those of considered groups. On the off chance that we see the enhanced geometries of Tc and Pd doped germanium confine clusters, we found that both structure are impeccable hexagonal prism closed shell structure. It can likewise be finished up by the figure that there is $\pi-\pi$ bond arrangement at the focal point of the HP ring and $\sigma$ bond is framing at the ring side means among the germanium atoms. So also, in the Pd doped germanium cluster display 58 valence

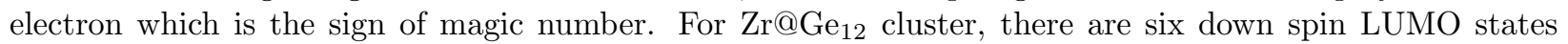
of $\mathrm{Ge}_{12}$ confine that impeccably connect covalently with valence state electrons which is fundamentally the same as past examination by Vijay kumar et al. ${ }^{[19]}$. For our case the 4 valence electrons $\left[4 \mathrm{~d}^{2} 5 \mathrm{~s}^{2}\right]$ of $\mathrm{Zr}$ totally share bonding with germanium enclosure and give huge HOMO-LUMO hole. It additionally shows that the HOMO and LUMO is roughly confined on the whole molecule. Because of this enormous energy gap, LUMO can scarcely gain electron from closed shell HOMO. So the enormous estimation of energy gap show lower reactivity in compound and photochemical procedure with electron move ${ }^{[59]}$. Based on above investigation, the cluster with huge energy can gap seen as building blocks of the novel materials.

In the next part of discussion, we describe the charge transfer mechanism by calculating the natural bond orbital analysis ${ }^{[43-44]}$. Since, among all the TM metals, studied in this work, only Pd (2.2) is more electronegative than germanium (2.01) on Pauling scale. It means the charge will always transfer from Pd to Ge atoms and in other TM atom; the charge will transfer from Ge atom to TM (Tc, Zr, and Co) atoms. The natural population analysis precisely determined the distribution of electrons in various sub shell of their atomic orbitals.

It is worth mentioning that the many body system properties like electronic geometry, dipole moment, polarizability are influenced by the atomic charges ${ }^{[60]}$. It can be seen from table 4 , the most electronegative charge of -1.883e accumulated for Tc, whereas all germanium atoms gained positive charge. The electrostatic point of view tells us that most electronegative atoms have tendency to donate electron and electropositive atoms have inclination to accept electrons, it means here charge is transferring from Tc atom to germanium cage. Similarly, Pd atom plays a donor role in $\mathrm{Pd} @ \mathrm{Ge}_{\mathbf{1 2}}$ cluster. In the $\mathrm{Zr}_{\mathrm{G}} \mathrm{Ge}_{\mathbf{1 2}}$ system, the charge accumulated by the $\mathrm{Zr}$ atom is -2.871 which again donate the electrons to $\mathrm{Ge}_{\mathbf{1 2}}$ cage. The case of Co doped $\mathrm{Ge}_{\mathbf{1 2}}$ cage is quite different. Here the charge is transferring from $\mathrm{Ge}_{\mathbf{1 2}}$ cage to TM metal Co atom.

\section{Partial Density of states -}

In order to investigate the electronic properties, variation in the HOMO-LUMO gap and chemical bonding nature due to TM metal doping in $\mathrm{Ge}_{12}$ cage cluster, we have performed subtleties examination of the atomic orbital to examine the contribution of different TM's (s, p, or d) and Ge (s, p) orbital's by calculating partial density of states (PDOS). The DOS is acquired by utilizing GaussSum programming ${ }^{[41]}$. It very well may be seen that the partial density of states means the number of different states where the electrons are allowed to occupy at a particular energy level can be significantly change when the $\mathrm{Ge}_{12}$ cluster are doped with $\mathrm{Tc}$, $\mathrm{Pd}, \mathrm{Zr}$, and Co atoms. As shown in the figure 6, the density of states of pure $\mathrm{Ge}_{12}$ cluster, the position of spin up and spin down DOS are same but after the doping of TM's (Tc, Pd, Zr, and Co) the spin up and spin down states in DOS exhibit the presence of electronic polarization.

The PDOS provide basic analysis of the interaction between the TM's atom orbital's and germanium cage. The peak of spin up and spin down near the Fermi level define the localization domains and corresponds to chemically reactive regions. The charge transfer between the TM atoms and the germanium cage generate the electric field, on the basis of this charge transfer, polarization can be explained. Study of natural bond orbital analysis [Supplementary Information] explain that electronic states near the Fermi level mainly come from $4 \mathrm{~s}, 4 \mathrm{p}$ orbital's of germanium cage and $4 \mathrm{~d}$ orbital of TM atoms. As we can see in table 2 [SI] that there are $4 \mathrm{~s}^{1.60} 4 \mathrm{p}^{2.38}$ orbital contributions of Ge atom and $4 \mathrm{~d}^{9.55}$ orbital contribution of $\mathrm{Pd}$ atom are mainly dominated near the Fermi level of $\mathrm{Ge}_{12} \mathrm{Pd}$ system. These all contribution has shown in alpha dos in the PDOS figure 6 and the p-d hybridization is mostly responsible for localized electrons near the Fermi level. Similarly, In $\mathrm{Ge}_{12} \mathrm{Tc}$, there is $4 \mathrm{~s}^{1.55}, 4 \mathrm{p}^{2.30}$ orbital of Ge atom $4 \mathrm{~d}^{7.63}$ orbital of Tc atom are contributing near the Fermi level and it is also clear from the PDOS. 


\section{Conclusions}

In this present report, we analyzed the stability and electronic properties of $\mathrm{M} @ \mathrm{Ge}_{12}(\mathrm{M}=\mathrm{Co}, \mathrm{Pd}, \mathrm{Tc}$, and $\mathrm{Zr}$ ) nanoclusters using the density functional theory. The outcomes are as follows:

1. We can identify that the $\mathrm{D}_{6 \mathrm{~h}}$ symmetry hexagonal prism structure is unique for germanium as well as silicon cage as described by José M. Goicoechea in his research ${ }^{[58]}$. It can be seen by figure 2 , the HP, Ih, and HAP structure reach their maximum stability when total valence electron in the system count 52-58 electrons. In this case TM atoms completely fall into the germanium cage.

2. The magnitude of binding energy of the clusters indicate that the doping of $4 \mathrm{~d}$ transition metal Tc, and Zr gives most stable structure rather than $3 \mathrm{~d}$ transition metal Co atom.

3. The results show that doping of $4 \mathrm{~d}$ transition metal like $\mathrm{Tc}, \mathrm{Zr}$, and $\mathrm{Pd}$ have relatively large gap in compare to 3d transition metal Co. Here we obtain $1.96 \mathrm{eV}\left[\mathrm{Tc} @ \mathrm{Ge}_{12}\right], 1.96 \mathrm{eV}\left[\mathrm{Pd} @ \mathrm{Ge}_{12}\right], 1.86 \mathrm{eV}\left[\mathrm{Zr}_{\mathrm{Ge}_{12}}\right]$, and $0.97 \mathrm{eV}\left[\mathrm{Co} @ \mathrm{Ge}_{12}\right]$ HOMO-LUMO gap for the most stable clusters. The stability of these clusters can be defined using closed shell electronic configuration and the contribution of $\pi$ and $\sigma$ bond.

4. Charge transfer mechanism shows that the $\mathrm{Tc}, \mathrm{Pd}$ and $\mathrm{Zr}$ atoms play role as a electron donor in the system whereas Co inclined to accept the electrons.

5. PDOS calculation provides the information of localized "d" electrons near the Fermi level. The electron density is mainly distributed around the TM atoms. PdGe 12 would be a good candidate as the building block with high magnetic moment for cluster assembly system.

So the overall conclusions suggest that the investigations of new hybrid semiconductor clusters doped with different TM atoms are very useful in electronic devices, laser application and sensors. The theoretical modeling also show possibility of designing miniature devices using pure and TM doped hybrid semiconductor clusters.

\section{Acknowledgements}

Complete calculations are done by using the Gaussian software facility at Indian Institute of Technology, Bombay. We would also like to thanks Prof. Alok Shukla for providing this facility.

\section{Author Contributions}

R. Trivedi and Vikash Mishra conceived the idea and performed the calculations.

\section{Compliance with ethical standards:}

Conflict of interest: The authors declare that they do not have any conflict of interest.

\section{Data availability statement -}

The raw/processed data required to reproduce these findings cannot be shared at this time as the data also forms part of an ongoing study.

\section{References -}

[1] D. Bandyopadhyay, Structural Chemistry . 2019 ,30 , 955-963.

[2] R. Trivedi, D. Bandyopadhyay, Journal of Material science.2019 , 54 , 515-528.

[3] R. Trivedi, D. Bandyopadhyay Journal of Material science .2018 , 53 , 8263-8273.

[4] R. Trivedi R, K. Dhaka, D. Bandyopadhyay, RSC Advance .2014 , 4 , 64825- 64834.

[5] P. Claes, V. T. Ngan, M. Haertelt, J. T. Lyon, A. Fielicke, M.T. Nguyen, P. Lievens, E. Janssens, J. Chem. Phys . $2013,138,194301$.

[6] X. Huang, H.G. Xu, S. Lu, Y. Su, R. B. King, J. Zhao, W. Zheng,Nanoscale . 2014 , 6 , 14617-14621. 
[7] K. Dhaka, D. Bandyopadhyay, RSC Advance . 2015 ,5 , 83004-83012.

[8] Y. Jin, Y. Tian, Y. Kuang, C. Lu, J. L. Cabellos, S. Mondal, G. Merino, J. Phys. Chem. C. 2016 , 120 , 8399-8404

[9] J. Atobe, K. Koyasu, S. Furusea, A. Nakajima, PCCP.2012 , 14 , 9403-9410.

[10] L. N. Pham, M. T. Nguyen, J. Phys. Chem. A.2017, 121 , 1940-1949.

[11] A. A. Saranin, A. V. Zotov, V. G. Kotlyar, T. V. Kasyanova, O. A. Utas, H. Okado, M. Katayama, K. Oura, Nano Lett.2004 , 4 , 1469.

[12] K. Dhaka, D. Bandyopadhyay, RSC Advance, 2015 ,5 , 83004-83012.

[13] Y. Kamata, Mater. Today 2008 , 11, 30-38.

[14] R. Pillarisetty, Nature . 2011 , 479 , 324-328.

[15] D. Bandyopadhyay, J. Mol. Model . 2012 ,18 , 3887-3902.

[16] M. Kumar, N. Bhattacharyya, D. Bandyopadhyay, J. Mol. Model . 2012 , 18 , 405-418.

[17] H. Xiaoming et al. J. Phys. Chem. C 2015 ,119, 20, 10987-10994.

[18] H. G. Xu, X. Y. Kong, X. J. Deng, Z. G. Zhang, W. J. Zheng, J. Chem. Phys . 2014 , 140 , 024308.

[19] V.Kumar, Y. Kawazoe, Phys. Rev. Lett . 2002 ,88, 235504.

[20] J. Lu, S. Nagase, Chem. Phys. Lett . 2003 ,372 , 394-398.

[21] S. Mahtout, C. Siouani, S. Safer, F. Rabilloud, J Phys Chem A. 2018 , 122 , 662-677.

[22] N. A Borshch, S. I. Kurganskii, Inorg Mater .2018 , 54 , 1-7

[23] S. Neukermans, X. Wang, N. Veldeman, E. Janssens, R.E. Silverans, P. Lievens, Int. J. Mass Spectrom . 2006 ,252, 145-150.

[24] C. Tang, M. Liu, W. Zhu, K. Deng, Comp. Theor. Chem .,2011 , 56 , 969

[25] D. Bandyopadhyay, P. Sen.. J. Phys. Chem . A .2010, 114, 1835.

[26] N. Kapila, V. K. Jindhal, H. Sharma. Physica B ,2011 , 406 , 4612;

[27] J. Wang, L. Ma, J. Zhao, G. Wang, J. Phys.: Condens. Matter , 2008, 33 , 5223

[28] W. J. Zhao, Y. X. Wang, J. Mol. Struct. (THEOCHEM),2009 , 901 , 18

[29] V. Kumar, Y. Kawazoe, Applied Physics Letters ,2002 , 80 , 859-861.

[30] J. Lu, S. Nagase, Chemical Physics Letters ,2003, 372 , 394-398.

[31] K. Burke, J. P. Perdew et al. Recent Progress and New Directions . Plenum. 1998

[32] J. P. Perdew, 1991 . Akademie, Berlin

[33] A. D. Becke, Phys Rev A . 1998, $38,3098-3100$.

[34] C. Lee, W. Yang, R. G. Parr, Phys Rev B . 1988 ,37, 785-789.

[35] M. J. Frisch, G. W. Trucks, H. B. Schlegel, G. E. Scuseria, M. A. Robb, J. R. Cheeseman,V. G. Zakrzewski, J. A. Montgomery Jr, R. E. Stratmann, J. C. Burant, S. Dapprich, J. M. Millam, A. D. Daniels, K. N. Kudin, M. C. Strain, O. Farkas, J. Tomasi, V. Barone, M. Cossi, Cammi B. RMennucci, C. Pomelli, C. Adamo, S. Clifford, J. Ochterski, G. A. Petersson, P. Y. Ayala, Q. Cui, K. Morokuma, D. K. Malick, A. D. Rabuck, K. Raghavachari, J . B. Foresman, J. Cioslowski, J. V. Ortiz, A. G. Baboul, B. B. Stefanov, B. Liu, A. Liashenko, P. Piskorz, I. Komaromi, R. Gomperts, R. L. Martin, D. J. Fox, T. Keith, Al-LahamMA, 
C. Y. Peng, A. Nanayakkara, M. Challacombe, P. M. W. Gill, B. Johnson, W. Chen, M. W. Wong, J. L. Andres, C. Gonzalez, M. Head-Gordon, E. S. Replogle, J. A. Pople. 2004 Gaussian 03, revision E01 Gaussian, Wallingford

[36] J. Wang, G. J. Han, J Chem Phys. 2005 ,123 , 064306-064321.

[37] J. G. Han, F. Hagelberg, J Mol Struct THEOCHEM ,2001 , 549 , 165-180.

[38] P. Guo, Z. Y. Ren, F. Wang, J. Bian, J. G. Han, G. H. Wang, J Chem Phys . 2004, 121 , 12265-12275.

[39] L. J. Guo, X. Liu, G. F. Zhaoa, Luo Y. H. J. Chem Phys. 2007 , 126 , 234704-234710.

[40] Hui-Fang Li and Huai-Qian Wang, Probing the stability of neutral and anionic transition-metal-doped golden cage nanoclusters: $\mathrm{M} @ \mathrm{Au}_{16}(\mathrm{M}=\mathrm{Sc}, \mathrm{Ti}, \mathrm{V})$, Phys.Chem.Chem.Phys., 2014, 16, 244.

[41] N. M. Boyle, A. L. Tenderholt, K. M. Langner, J. Comp. Chem . 2008 , 29 , 839-845.

[42] A. E. Reed, F. Weinhold, J. Chem. Phys . 1985 ,83, 1736-1740.

[43] A. E. Reed, R. B. Weinstock, F. Weinhold, J. Chem. Phys .1985 , 83 , 735-746.

[44] U. Schwarz, T. Sophie, O. Janson, K. Cevriye, K. Cornelius, U. Burkhardt, H. Rosner, F. Steglich, Y. Grin. Angew. Chem. Int. Ed ., 2013 , 52 : 9853-9857.

[45] H. Wang, Y. G. Khait, M. R. Hoffmann, Molecular Physics ,2005 , 103 , 263-268.

[46] Q. Jing, F. Y. Tian, Y. X. Wang, J. Chem. Phys .2008 , 128 , 124319.

[47] M. Lasmi, S. Mahtout, F. Rabilloud, Computational \& Theoretical Chemistry, Computational and Theoretical Chemistry,2020 , $1181,112830$.

[48] Z. Tan, T. Zhou, Y. Yang, AIP Advances, 2016 ,6 , 125123.

[49] S. Jaiswal, V. Kumar, Computational \& Theoretical Chemistry, 2015 , 1075 , 87-97.

[50] M. Brack, Rev. Mod. Phys . 1993, 65, 677-732.

[51] I. Langmuir, Science, 1921, 54, 59-67.

[52] W. D. Knight, K. Clemenger, W.A. de Heer, W.A. Saunders, M. Y. Chou, M. L. Cohen, Phys. Rev. Lett . 1984, 52, 2141-2143.

[53] P. Pyykko, J. Organomet. Chem . 2006 ,691 , 4336-4340.

[54] M. B. Abreu, A. C. Reber, S. N. Khanna, J. Phys. Chem. Lett . 2014, 5 , 3492-3496

[55] S. A. Claridge, A. W. Castleman, S. N. Khanna, C. B. Murray, A. Sen, P. S. Weiss, ACS Nano, 2009 , 3, 244-255

[56] V. Kumar, Y. Kawazoe, Appl. Phys. Lett . 2003 ,83 , 2677.

[57] L. Tian, C. Feiwu, J. Comput. Chem ., 2012 ,33 , 580-592.

[58] J. M. Goicoechea, J. E. McGrady, Dalton Trans ,2015, 44 , 6755.

[59] H. Abou-Rachid, Y. Song, A. Hu, S. Dudiy, S. V. Zybin, W. A.Goddard, J. Phys. Chem. A . 2008 , 112 (46), 11914-11920.

[60] I. Sidis, Y. G. Sidir, M. Kumalar, E. Tasal, J. Mol. Struct . 2010 , 964, 134-151.

\section{Tables -}

Table 1 Bond length $(\AA)$, Frequency $\left(\mathrm{cm}^{-1}\right)$, and Ionization potential $(\mathrm{eV})$ of different dimmers, comparison with theoretical and experimental data's 
Table 2 Bond length $(\AA)$, and Frequency $\left(\mathrm{cm}^{-1}\right)$, of different dimmers with different functional

Table 3 Calculation of the average binding energy, HOMO-LUMO gap, bond length of Ge-TM, and the relative energy $(\Delta \mathrm{E})$ difference between stable isomers

Table 4 Accumulation of natural charges in $\mathrm{TM} @ \mathrm{Ge}_{12}[\mathrm{TM}=\mathrm{Co}, \mathrm{Pd}$, Tc, and $\mathrm{Zr}]$ clusters

Figures -

Figure 1 . Optimized minimum energy structure of pure $\mathrm{Ge}_{12}$ cluster with high symmetry points. Arrows show endohedral and exohedral position.

Figure 2. Trends of decreasing stability order in optimized structure of $\mathrm{M}_{\mathrm{G}} \mathrm{Ge}_{12}(\mathrm{M}=\mathrm{Co}, \mathrm{Pd}$, Tc, and $\mathrm{Zr}$ ) with minimum energy difference among them.

Figure 3 . The average binding energy of $\mathrm{TM} @ \mathrm{Ge}_{12}[\mathrm{TM}=\mathrm{Co}, \mathrm{Pd}, \mathrm{Tc}$, and $\mathrm{Zr}]$ clusters compared with pure $\mathrm{Ge}_{12}$ cluster with their minimum energy structure. Blue band shows the binding energy of pure germanium cage cluster.

Figure 4. Calculated binding energy of $\mathrm{TM} @ \mathrm{Ge}_{12}[\mathrm{TM}=\mathrm{Co}, \mathrm{Pd}, \mathrm{Tc}$, and $\mathrm{Zr}]$ with all other high key point symmetry.

Figure 5 . HOMO-LUMO gap of TM@Ge 12 [TM = Co, Pd, Tc, and $\mathrm{Zr}]$ clusters

Figure 6 Partial densities of states (PDOS) for $\mathrm{Ge}_{12}$ and $\mathrm{Ge}_{12} \mathrm{Pd}, \mathrm{Ge}_{12} \mathrm{Tc}$ shows a significant change in the PDOS at the Fermi level due to doping of Pd and Tc.

\section{Hosted file}

Figures.docx available at https://authorea.com/users/353662/articles/477458-exploring-thestructural-stability-order-and-electronic-properties-of-transition-metal-m-ge12-m-co-pdtc-and-zr-doped-germanium-cage-clusters

\section{Hosted file}

Table_file.docx available at https://authorea.com/users/353662/articles/477458-exploringthe-structural-stability-order-and-electronic-properties-of-transition-metal-m-ge12-mco-pd-tc-and-zr-doped-germanium-cage-clusters 